\title{
Oral Rivaroxaban in Symptomatic Deep Vein Thrombosis
}

\author{
Ahmed Farhan1, Maliha Bukhari1, Junaid Umar² and Mudassar Ali Raza1 \\ Department of General Medicinel / Radiology², Pakistan Institute of Medical Sciences (PIMS), Islamabad, Pakistan
}

\begin{abstract}
Objective: To evaluate the efficacy of oral rivaroxaban compared to warfarin in patients with deep vein thrombosis (DVT). Study Design: Open label randomized controlled study.

Place and Duration of Study: Department of General Medicine, Pakistan Institute of Medical Sciences (PIMS), Islamabad from January 2016 to January 2018.

Methodology: Patients of both genders between 18 and 60 years of age with Doppler ultrasound-confirmed DVT were included in the study. Pregnant patients and those with advanced liver, renal disease, those with a previous history of DVT, malignancy, with a platelets count of less than 50000/ul were excluded from the study. Rivaroxaban was given in a dose of $15 \mathrm{mg}$ twice daily for 21 days followed by $20 \mathrm{mg}$ once daily. Patients in the warfarin group were given heparin for 3 to 5 days followed by warfarin for 3 to 6 months. The primary efficacy outcome was patency of the vessel at 3 and 6 months of treatment. The principal safety outcomes were major and minor bleeding during the study period.

Results: A total of 151 patients with acute symptomatic deep vein thrombosis were enrolled in the study. Half of the patients were given warfarin and the other half rivaroxaban for 6 months. At three months, there were no significant differences observed in vessel patency in the rivaroxaban group (22.4\%) as compared to warfarin group (26.7\%) but after 6 months of therapy, vessel patency was significantly more in the rivaroxaban group. Adverse events did not show any significant differences

Conclusion: Rivaroxaban had an efficacy superior to warfarin in terms of vessel patency after six months of therapy but adverse events were similar in both the groups
\end{abstract}

Key Words: Rivaroxaban, Deep vein thrombosis, Warfarin, Factor Xa inhibitors, Vitamin K antagonists.

How to cite this article: Farhan A, Bukhari M, Umar J, Raza MA. Oral rivaroxaban in symptomatic deep vein thrombosis. $J$ Coll Physicians Surg Pak 2019; 29(9):814-8.

\section{INTRODUCTION}

Venous thromboembolism is the third most common cause of vascular death after myocardial infarction and stroke. ${ }^{1}$ It has an annual incidence of approximately 1 or 2 cases per 1000 persons in the general population. ${ }^{2}$ The exact incidence and prevalence of deep-vein thrombosis in Pakistan is not known. The prevalence of DVT in Pakistani patients with swollen legs and those with paraplegia due to spinal cord pathology was $51.9 \%$ and $2.7 \%$, respectively. 3,4 Heparin followed by warfarin was considered as the gold standard treatment in patients with DVT, probably because it was the only medical treatment available. However, treatment with vitamin $\mathrm{K}$ antagonists poses risks of bleeding ranging from minor to serious bleeds requiring transfusions. The novel oral anticoagulants like rivaroxaban do not require regular laboratory monitoring, have few drug interactions and are considered safe for continuation on long term basis. ${ }^{5}$ Studies have shown the non-inferiority and cost effectiveness of rivaroxaban compared to subcutaneous enoxaparin overlapped with warfarin (enoxaparin-VKA)

Correspondence to: Dr. Ahmed Farhan, MW-6, Department of

General Medicine, Pakistan Institute of Medical Sciences

(PIMS), Islamabad, Pakistan

E-mail:drfarhan992@gmail.com

Received: August 08, 2018; Revised: April 18, 2019;

Accepted: May 03, 2019 with regard to the incidence of recurrent venous thromboembolism. 6,7

Rivaroxaban is fast acting, available as an oral formulation, has a more predictable response and very few drug interactions compared to warfarin. It reversibly inhibits both the intrinsic and extrinsic pathways and has an oral bioavailability of 80 to $100 \%$. Having said that, disadvantages of rivaroxaban include a lack of availability of the antidote andexanet alfa in Pakistan, lack of efficacy in obese individuals ${ }^{8}$, inability to monitor rivaroxaban activity, and a higher cost of the medicine.

In developing countries like Pakistan, treatment of deep vein thrombosis requires physicians' attention at multiple levels as laboratory facilities are nonexistent, dietary habits are limited to vegetable intake and tea, and the prevailing herbal and homeopathic practices affect the efficacy of warfarin.9,10 Cost of the medications and periodic INR monitoring is also one of the strong factors leading to nonadherence. Patients also need hospitalisation for heparin therapy which constitutes an economic burden at government level as well. Local studies on rivaroxaban have not been carried out and little is known about its efficacy in Pakistani patients with deep vein thrombosis.

The objective of this study was to evaluate the efficacy of oral rivaroxaban compared to warfarin in patients with DVT. 


\section{METHODOLOGY}

This open label randomised interventional study was conducted in the Department of General Medicine, Pakistan Institute of Medical Sciences (PIMS), Islamabad from January 2016 to January 2018 to compare the efficacy of rivaroxaban with warfarin in patients with DVT. Hospital Ethical Review Board approval was obtained before the start of study and written informed consent was taken from all patients prior to enrollment in the study.

Patients of both genders between 18 and 60 years of age with DVT confirmed on Doppler ultrasound, were included in the study after a written informed consent was obtained. Patients with a previous history of DVT or post-phlebitis syndrome were excluded. Patients with advanced liver disease (those with ascites, varices, portal hypertension and bilirubin of $2 \mathrm{mg} / \mathrm{dl}$ or more), or liver disease with abnormal liver synthetic function (baseline prothrombin time of greater than 6 seconds and albumin levels of less than $2.5 \mathrm{mg} / \mathrm{dl}$ ), patients with renal disease with a creatinine of greater than $3 \mathrm{mg} / \mathrm{dl}$ or GFR of less than $30 \mathrm{ml} / \mathrm{min}$, patients with underlying malignancy, those with a platelets count of less than $50000 / u l$, pregnant patients and patients with a positive thrombophilia screen were also excluded from the study.

Patients were randomised into two groups by the android mobile software "statistics and sample size version 10". Only the investigator performing Doppler/duplex ultrasound was blinded. Two investigators collected the data and recorded their findings on the questionnaire.

The study group was given oral rivaroxaban $\left(\mathrm{xcept}^{R}\right)$ at doses of $15 \mathrm{mg}$ twice daily for three weeks followed by $20 \mathrm{mg}$ once daily, while the control group was given conventional heparin $7500 \mathrm{IU}$ subcutaneous QID for 3 to 5 days along with warfarin $10 \mathrm{mg}$ once daily for two days followed by warfarin $5 \mathrm{mg}$ once daily for 6 months.

Patients were followed for 6 months at three weekly intervals. All patients were given the study medications for three weeks after which they were to follow with fresh $A L T$, creatinine and INR. A predesigned questionnaire was filled by the investigators focusing on compliance, any adverse effects especially any bleeding episodes occurring during the preceding three weeks' interval. Patients were also given emergency contact number to report in case any adverse event occurs.

Repeated Doppler ultrasound was done after 3 and 6 months for evaluation of the presence of thrombus in deep veins. For secondary outcome, patients were interviewed at three-weekly intervals for any adverse events.

The primary efficacy outcome was vessel patency as determined by Doppler and duplex ultrasound at the end of three and six months. Parameters determining vessel patency included clot lysis and present or absence of blood flow.

Secondary safety outcomes included any major or minor bleed during the study period. Major bleeding was defined as clinically overt bleeding leading to a decrease in hemoglobin of $2 \mathrm{gm} / \mathrm{dl}$, requiring red cell transfusion of one or more pints and/or bleeding at critical sites like intracranial and retroperitoneal bleeding. Minor bleeding was defined as clinically overt bleeding that did not meet the criteria for major bleeding but was associated with medical intervention, unscheduled contact with a physician, interruption or discontinuation of the study drug, or discomfort or impairment of activities of daily life.

SPSS version 16 was used for analysing the results. Frequencies and percentages were performed for gender and categorical data. Chi-square test was performed for qualitative tests. Independent and paired t-tests were performed for quantitative variables. Logistic regression analysis was used to predict outcomes. $\mathrm{P}$-value of 0.05 or less was considered as significant.

\section{RESULTS}

A total of 183 patients were initially enrolled in the study but 32 patients were lost to follow up, 20 in the warfarin group and 12 in the rivaroxaban group. The remaining 151 patients with deep vein thrombosis were grouped into two, half of them (75) in the warfarin group while the other half (76) in the rivaroxaban group. Eighty-five $(56.3 \%)$ patients comprised of females. Most patients had combined popliteal and femoral vein thrombosis, followed by popliteal vein and combined popliteal, femoral and iliac vein thrombosis. The mean age of the patients was $34.99 \pm 11.74$ std. Among patients who were on warfarin, the mean INR was $2.09 \pm 0.77$. Fortyone $(54.67 \%)$ pateints had INR below the target range of 2.0 , while $10(13.33 \%)$ patients had INR above the target range of 3.0. Only $24(32 \%)$ patients had their mean INR within the target range of 2.0 to 3.0 . The presence of thrombus was documented in $114(75.5 \%)$ and 36 $(23.8 \%)$ patients after three and six months, respectively.

After 3 months of treatment, vessel patency was demonstrated in $17(22.4 \%)$ and $20(26.7 \%)$ patients in the rivaroxaban and warfarin group, respectively. Vessel patency was not significantly different in the rivaroxaban and warfarin group after 3 months of therapy (odds ratio $=0.896,95 \% \mathrm{Cl}=0.394-1.860, \mathrm{p}=0.575)$. After six months of treatment, vessel patency occurred in 64 $(84.2 \%)$ and $51(68 \%)$ in the rivaroxaban and warfarin group, respectively. Vessel patency occurred significantly more in patients in the rivaroxaban group compared to warfarin $(p=0.02$, odds ratio $2.5,95 \% \mathrm{Cl}=1.145-5.5)$. Among patients in the warfarin group, vessel patency was seen in significantly less number of patients who had subnormal INR. 
After excluding patients who had a sub-therapeutic INR, a total of 34 patients were found, with INR in the therapeutic range $>2.0$. Comparing patients on rivaroxaban and those with an INR greater than 2, there was a significant difference in the efficacies of the two studied drugs at three months of treatment in favour of warfarin. Fifteen out of 34 , i.e. $44.12 \%$ had vessel patency in warfarin group compared to 17 out of 76 , i.e. $(22.37 \%)$ in the rivaroxaban group $(p=0.02)$. This difference was not significant after 6 months of therapy with 29 out of 34, i.e. $(85.29 \%)$ vessel patency in warfarin group compared to 64 out of $76(84.21 \%)$ in the rivaroxaban group at six months of therapy $(p=0.88)$.

Major bleeding occurred only in 2 cases, both of which belonged to the warfarin group and had INR above the therapeutic range. Minor bleeds occurred in a total of 7 patients, 4 in the rivaroxaban group and 3 in the warfarin group. There were significant changes seen in ALT and creatinine. ALT increased from a mean value of $33.5 \mathrm{U} / \mathrm{I}$ and $32.3 \mathrm{U} / /$ to $43.4 \mathrm{U} / /$ and $34.5 \mathrm{U} / /$ in the rivaroxaban and warfarin group, respectively. Creatinine values decreased in both groups significantly from mean 0.77 $\mathrm{mg} / \mathrm{dl}$ and $0.92 \mathrm{mg} / \mathrm{dl}$ to $0.56 \mathrm{mg} / \mathrm{dl}$ and $0.58 \mathrm{mg} / \mathrm{dl}$ in the rivaroxaban and warfarin group, respectively. Results of the study are summarised in Table I.

Table I: Summary of results of the study.

\begin{tabular}{l|c|c|c}
\hline & $\begin{array}{c}\text { Rivaroxaban } \\
\text { no }(\%)\end{array}$ & $\begin{array}{c}\text { Warfarin } \\
\text { no }(\%)\end{array}$ & p-value \\
\hline Male sex & $44(57.9)$ & $22(29.3)$ & \\
\hline Mean age (years) & $37.05+/-10.35$ & $32.91+/-12.72$ & \\
\hline Dvt & $24(31.58)$ & $8(10.67)$ & \\
Popliteal & $8(10.52)$ & $15(20)$ & \\
Femoral & $4(5.26)$ & $0(0)$ & \\
Iliac & $40(52.63)$ & $31(41.33)$ & \\
Popliteal + femoral & 0 & $21(28)$ & \\
Popliteal + femoral + iliac & & & 0.57 \\
\hline Thrombus at 3 months & $17(22.37)$ & $20(26.67)$ & \\
Absent (\%) & $59(77.63)$ & $55(73.33)$ & \\
Present (\%) & & & 0.02 \\
Thrombus at 6 months & $64(84.21)$ & $51(68)$ & \\
Absent (\%) & $12(15.79)$ & $24(32)$ & \\
Present (\%) & & & \\
\hline ALT & $33.47 \pm 20$ & $32.29 \pm 25$ & 0.749 \\
Baseline & $43.40 \pm 17$ & $34.5 \pm 3$ & 0.008 \\
After treatment & & & \\
\hline Creatinine & $0.78 \pm 0.19$ & $0.92 \pm 0.33$ & 0.002 \\
Baseline & $0.56 \pm 0.31$ & $0.58 \pm 0.30$ & 0.805 \\
\hline After treatment & $0(0)$ & $2(2.7)$ & 0.15 \\
\hline Major bleed & $4(5.3)$ & $3(4)$ & 0.71 \\
\hline Minor bleed & $72(94.7)$ & $70(93.3)$ & 0.72 \\
\hline No bleed & & &
\end{tabular}

\section{DISCUSSION}

Our study compared rivaroxaban with warfarin in patients with DVT. The mean age of the pateints were 32 years and 37 years in the warfarin and rivaroxaban group, respectively. The mean ages ranged from 55 years to 65 years in most studies.6,11 Although, rivaroxaban has been studied in different age-groups and age does not influence its efficacy, pharmacokinetics or pharmacodynamics. ${ }^{12}$ The manufacturer recommends using the drug with caution in geriatric patients. Almost half of the patients $(54.6 \%)$ had subtherapeutic mean INR. The percentages of subtherapeutic INR value vary ranging from $23 \%$ to $43 \% .6,13$ In a study on anticoagulation in patients with prosthetic heart valve replacement in Pakistani patients, INR was therapeutic in the range of 2.0 to 3.0 in $68.85 \%$ of the patients. ${ }^{14}$ The high percentage of patients with subtherapeutic INR in this study could have multiple reasons ranging from as simple as dietary habits to complex drug interactions. Various studies have demonstrated significant variations in plasma vitamin $\mathrm{K}$ levels and INR because of the variations in dietary habits, drug interactions and genetic infuences. ${ }^{15}$ Secondly, the study by Akhtar et al. 14 included patients with prosthetic valves which is considered as a serious problem by the patients in comparison to deep vein thrombosis. The present study did not investigate the causes of sub-therapeutic INR in our patient population. However, immobilisation due to comorbid conditions like trauma/fracture was found as the most common cause contributing to venous thrombosis in one study. ${ }^{16}$

It was found that after six months of therapy, rivaroxaban was more efficacious than warfarin and with a better safety profile as well. Studies have proven the efficacy of rivaroxaban in patients undergoing hip surgery ${ }^{17}$ and non-valvular atrial fibrillation.18 The efficacy of rivaroxaban was similar to warfarin after three months of therapy. In a subgroup of patients on warfarin whose INR was above 2, warfarin was found to be significantly more effective after 3 months but had similar efficacy after six months of therapy when compared to rivaroxaban. Studies comparing the efficacy of different anticoagulants in the severity of stroke in pateints with non-valvular atrial fibrillation concluded that standard dose NOACs or warfarin with a therapeutic intensity were both associated with a mild stroke. ${ }^{19}$ Guidelines recommend treatment of DVT for three months, especially patients who have provoked DVT and are at low risk of recurrence, a high percentage of patients had persistent thrombus at three months compared to 6 months of therapy. In the Einstein continued treatment study, treatment with rivaroxaban after 6 to 12 months of therapy prevented the rate of recurrence of DVT by $82 \%$ with a small risk of major hemorrhage. ${ }^{6} \mathrm{~A}$ study by Bawa et al. concluded that pateints treated with rivaroxaban compared to warfarin had less chances of developing DVT when followed 6 months after TKA and THA. ${ }^{20}$

A total of 9 events of bleeding occurred in this study; four minor bleeding events occurred in the rivaroxaban 
group, while five occurred in the warfarin group, including two major bleeding events requiring transfusion of blood products. The safety outcomes in both groups were similar in these patients. The Einstein and Xiala studies have similarly found no clinical difference in both the agents as far as adverse events were concerned rather patients on rivaroxaban were found to have less bleeding tendencies and thus had a better safety profile.21 The Einstein study although observed a significantly greater net clinical benefit with rivaroxaban as compared to warfarin. 6

ALT levels showed a slight elevation from baseline in both groups but significantly more so in the rivaroxaban group.

Literature is sparse on hepatotoxicity due to rivaroxaban; and trials have variable observations. In one study, the risk of hepatotoxicity was observed in $2.3 \%$ patients who were treated with rivaroxaban after orthopedic surgery. ${ }^{22}$ A number of case reports have been published demonstrating rivaroxaban-induced hepatoxicity. A systemic search of case reports on the Medline database was performed. Half of the patients developed hepatocellular pattern of liver injury, one-third developed cholestatis and another third developed a mixed pattern of liver injury with the use of rivaroxaban. Contrary to ALT levels, creatinine levels dropped significantly in both groups more so in the warfarin group. A nationwide retrospective cohort study evaluated the incidence of acute kidney injury in patients on the novel oral anticoagulants including rivaroxaban and warfarin. The study concluded that all the three NOACs were associated with a lower risk of acute kidney injury than warfarin. 23

Despite the benefits of rivaroxaban, warfarin is still the drug of choice in patients with renal disease. Furthermore, obese patients weighing greater than $120 \mathrm{Kgs}$ and those with a BMI of more than $40 \mathrm{Kg} / \mathrm{m}^{2}$ should avoid using rivaroxaban. Limited data is available regarding the use of NOACs in obese individuals. Case reports and smaller studies have got variable results. The case report of a middle-aged obese female, who developed pulmonary embolism despite on therapeutic dose of rivaroxaban, questions the efficacy of rivaroxaban in this subgroup of patients. 24

Similarly, LMWH is still considered superior in patients with malignancies. However, recent studies suggest that the novel oral anticoagulants have a similar efficacy and are better tolerated in patients with cancers. ${ }^{25}$

Less number of patients, use of a preparation manufactured by a national company rather than the original brand, relatively stable and young patients (without comorbid risks, malignancies, renal and hepatic diseases), and outcomes which were relatively subjective (vessel patency, thrombus and flow) were some of the limitations of this study.

\section{CONCLUSION}

Rivaroxaban was found to be superior to warfarin in clot resolution after six months of therapy, but had a similar efficacy after three months of therapy. Safety profile of both the agents were similar.

\section{ETHICAL APPROVAL:}

Hospital Ethical Review Board approval was obtained before the start of study.

\section{PATIENTS' CONSENT:}

Informed consent was taken from all patients prior to enrollment in the study.

\section{CONFLICTS OF INTEREST:}

No author has declared any sort of financial or other kind of support from anybody.

\section{AUTHORS' CONTRIBUTION:}

AF: Planned and designed the study, analysed the results of the study, wrote the discussion and reviewed the final draft.

MB: Collected data from the patient, wrote introduction and reviewed the final draft.

JU: Performed Doppler ultrasound and helped in analysing data.

MAR: Collected data and reviewed the final draft.

\section{REFERENCES}

1. Weitz J, Lensing A, Prins M, Bauersachs R, Westendorf J, Bounameaux $\mathrm{H}$, et al. Rivaroxaban or aspirin for extended treatment of venous thromboembolism. N Engl J Med 2017; 376:1211-22.

2. Spencer FA, Emery C, Lessard D. The worcester venous thromboembolism study: A population-based study of the clinical epidemiology of venous thromboembolism. J Gen Intern Med 2006; 21:722-7.

3. Hadi NU, Iqbal M, Awan $\mathrm{KH}$. Frequency of different causes of painful swollen leg in patients referred for sonographic examination at a teaching hospital. J Postgrad Med Inst 2016; 30:2.

4. Arsh A, Darain H, llyas SM. Epidemiology and prevalence of deep venous thrombosis in patients with spinal cord injury. Pak J Neurol Sci 2016; 11:22-5.

5. Buller HR, Lensing AWA, Prins $\mathrm{MH}$. A dose-ranging study evaluating once-daily oral administration of the factor $\mathrm{Xa}$ inhibitor rivaroxaban in the treatment of patients with acute symptomatic deep vein thrombosis: The Einstein-DVT doseranging study. Blood 2008; 112:2242-7.

6. The EINSTEIN Investigators. Oral rivaroxaban for symptomatic venous thromboembolism. N Engl J Med 2010; 363:2499-510.

7. Burness $C B$, Perry CM. Rivaroxaban: A review of its use in the treatment of deep vein thrombosis or pulmonary embolism and the prevention of recurrent venous thromboembolism. Drugs 2014; 74:243-62.

8. Martin K, Beyer-Westendorf J, Davidson BL, Huisman MV, Sandset PM, Moll S. Use of the direct oral anticoagulants in 
obese patients: Guidance from the SSC of the ISTH. J Thromb Haemost 2016; 14:1308-13.

9. Izzo AA, Ernst E. Interactions between herbal medicines and prescribed drugs. Drugs 2009; 69:1777-98.

10. Ge B, Zhang Z, Zuo Z. Updates on the clinical evidenced herbwarfarin interactions. Evid Based Complement Alternat Med 2014; 2014:957362.

11. Margolis JM, Deitelzweig S, Kline J, Tran O, Smith DM, Bookhart B, et al. Shorter hospital stays and lower costs for rivaroxaban compared with warfarin for venous thrombosis admissions. J Am Heart Assoc 2016; 5:e003788.

12. Moore KT, Wong P, Zhang L, Pan G, Foody J. Influence of age on the pharmacokinetics, pharmacodynamics, efficacy, and safety of rivaroxaban. Curr Med Res Opin 2018; 34:2053-61.

13. Chitsike RS, Rodger MA, Kovacs MJ, Betancourt MT, Wells PS, Anderson DR, et al. Risk of post-thrombotic syndrome after subtherapeutic warfarin anticoagulation for a first unprovoked deep vein thrombosis: Results from the reverse study. J Thromb Haemost 2012; 10:2039-44.

14. Akhtar RP, Abid AR, Zafar H, Khan JS. Aniticoagulation in patients following prosthetic heart valve replacement. Ann Thorac Cardiovasc Surg 2009; 15:10-7.

15. Proietti M, Hijazi Z, Andersson U, Connolly SJ, Eikelboom JW, Ezekowitz MD, et al. Comparison of bleeding risk scores in patients with atrial fibrillation: Insights from the RE-LY trial. J Intern Med 2018; 283:282-92.

16. Dabadghao VS, Diwan AG. A study of etiological and clinical profile with associated risk factors of deep vein thrombosis detected in a hospital based setting and review of literature of this as an emerging health problem in hospitals. Nat $J$ Integr Res Med 2018; 9:2.

17. Jiang S, Du L, Ni C. Comparing the efficacy, safety and cost of the anticoagulants: Rivaroxaban and nadroparin in hip replacement surgery. Int J Pharmacol 2018; 14:1-8.
18. Uchiyama S, Atarashi H, Inoue H, Kitazono T, Yamashita T, Shimizu W, et al. Primary and secondary prevention of stroke and systemic embolism with rivaroxaban in patients with non-valvular atrial fibrillation. Heart and vessels 2019; 34: 141-50.

19. Jung YH, Choi HY, Lee KY, Cheon K, Han SW, Park JH, et al. Stroke severity in patients on non-vitamin $\mathrm{K}$ antagonist oral anticoagulants with a standard or insufficient dose. Thromb Haemost 2018; 118:2145-51.

20. Bawa H, Weick JW, Dirschl DR, Luu HH. Trends in deep vein thrombosis prophylaxis and deep vein thrombosis rates after total hip and knee arthroplasty. J Am Acad Orthopaed Surg 2018; 26:698-705.

21. Ageno W, Mantovani LG, Haas S, Kreutz R, Monje D, Schneider J, et al. Safety and effectiveness of oral rivaroxaban versus standard anticoagulation for the treatment of symptomatic deep-vein thrombosis (XALIA): An international, prospective, non-interventional study. Lancet Haematol 2016; 3:e12-21.

22. Ageno W. Rivaroxaban: An evaluation of its cardiovascular benefit-risk profile across indications based on numbers needed to treat or harm, and on clinically meaningful endpoint comparisons. Drugs 2015;15:295-306.

23. Chan YH, Yeh YH, Hsieh MY, Chang CY, Tu HT, Chang SH, et al. The risk of acute kidney injury in Asians treated with apixaban, rivaroxaban, dabigatran, or warfarin for non-valvular atrial fibrillation: A nationwide cohort study in Taiwan. Int $\mathrm{J}$ Cardiol 2018; 265:83-9.

24. Schuh T, Stöllberger C. Pulmonary embolism despite rivaroxaban in an obese patient. Dtsch Med Wochenschr 2017; 142:1548-51.

25. Xiang E, Ahuja T, Raco V, Cirrone F, Green D, Papadopoulos J. Anticoagulation prescribing patterns in patients with cancer. J Thromb Thrombolysis 2018; 45:89-98. 